Journal of Advanced Research in Applied Mechanics

Journal homepage: www.akademiabaru.com/aram.html ISSN: 2289-7895

\title{
Preparation Methods and Thermal Performance of Hybrid Nanofluids
}

\author{
Nor Azwadi Che Sidik ${ }^{1, *}$, Idris M. Adamu², Muhammad Mahmud Jamil ${ }^{3}$ \\ 1 Malaysia - Japan International Institute of Technology (MJIIT) , Universiti Teknologi Malaysia, Jalan Sultan Yahya Petra, 54100 Kuala Lumpur, \\ Malaysia \\ School of Mechanical Engineering, Faculty of Engineering, Universiti Teknologi Malaysia, 81310 Skudai Johor, Malaysia \\ Department of Mechanical Engineering, Nigerian Defence Academy Kaduna, Nigeria
}

\section{ABSTRACT}

Hybrid nanofluid is a new nanotechnology fluid that is synthesized by dispersing two different nanoparticles into conventional heat transfer fluid. Recently, researchers have indicated that hybrid nanofluids can effectively substitute the convectional coolant especially those working at very high temperatures. In this paper a comprehensive literature on the synthesis of hybrid nanoparticles, hybrid nanofluid and thermal performance of hybrid nanofluid have been compiled and reviewed.

Keywords:

Hybrid nanofluid; thermal conductivity; nanofluid

Copyright $\odot 2020$ PENERBIT AKADEMIA BARU - All rights reserved

\section{Introduction}

It is clear from a survey of thermal properties that convectional heat transfer fluid such as water; ethylene glycol and oil possess low thermal conductivity compare to solid metals. In view of the above issue, a lot of research has been devoted in order to improve the thermal transport properties of the fluids. One of the possible techniques for improving heat transfer is by adding millimeter- or micrometer-sized particles in fluids. In recent years, nanofluids, coined by Choi [1], have been identified as an ideal candidate for enhancing heat transfer.

Nanofluids are new engineered fluid obtained by dispersing nanoparticles in a base fluid for the purpose of improving the thermal characteristics of the base fluid [2]. The nanofluid was observed to give better performance than that of convectional heat transfer fluid (oil, ethylene glycol, and water) [3]. Hybrid nanofluid as an extension of nanofluid is obtained by dispersing composite nano-powder or two different nanoparticles in the base fluid. It is believed that, hybrid nanofluid will offer good thermal characteristics as compared to the base fluid and nanofluid containing single nanoparticles as a result of synergistic effects [4]. Turco et al., [5] possibly the first who reported the synthesis of hybrid nano-composite particles, two different hybrids of PPY-CNT nano-composite and MWCNT on

\footnotetext{
* Corresponding author.

E-mail address: azwadi@utm.my 
magnetic $\mathrm{Fe}_{2} \mathrm{O}_{3}$ nanoparticles were studied. The thermal conductivity of hybrid nanofluid such as CNT-AuNP and CNT-CuNP shows decrease in enhancement compared to single nanoparticle due to compatibility effect of the nanoparticles [6]. Within the same year, Turco et al., [7] conducted a comprehensive experiment on the physiochemical properties of hybrid nanostructures for biotechnology application. Yen et al., [8] numerically investigated the effect of hybrid nanofluid in channel flows. In a different study, hybrid nano-polymer was prepared for the application in solar cell [9]. Synthesis characteristics of hybrid magnetic-polymer were investigated by TEM, HRTEM and magnetization measurements [10,11]. According to Jha and Ramaprabhu [12], a better enhancement in thermal conductivity was reported by hybridizing silver nano-particles with multi-walled carbon nano-tube. The influence of hybrid $\mathrm{Al}_{2} \mathrm{O}_{3}$ nanoparticle and micro encapsulated phase change material particles have shown a remarkable enhancement in terms of cooling effectiveness compared to single nanoparticles and water [13]. The finding of Suresh et al., [14] shows that $\mathrm{Al}_{2} \mathrm{O}_{3}-\mathrm{Cu}$ hybrid gives thermal conductivity enhancement of $12.11 \%$ at volume fraction of $2 \%$. However, the thermal conductivity and viscosity of the hybrid increases with increasing volume fraction but the increase is higher in terms of viscosity.

In a different study, Tessy and Sundara [15] synthesized a hybrid nanostructure (f-MWNT+f-HEG) by a post-mixing technique. Then, the hybrid nanostructure was used for synthesizing nanofluids by dispersing it in DI water and EG. The hybrid nanofluid was found to have $20 \%$ enhancement in thermal conductivity at volume fraction of $0.005 \%$. The maximum enhancement of the heat transfer coefficient was about $289 \%$ for a $0.01 \%$ volume fraction of $\mathrm{f}-\mathrm{MWNT}+\mathrm{f}-\mathrm{HEG}$ at Reynolds number of 15500 [15]. In addition, metal particles were observed to possess high thermal conductivity as a result of small inter-atomic space which helps in easy conduction [16].

Generally, thermal conductivity is a key parameter for heat transfer enhancement of the hybrid nanofluid. The thermal conductivity and heat transfer parameters of nanofluid depends on a number of factors, such as nanoparticles type, nanoparticles size, stability, base fluid type, fluid temperature etc [17-19]. Baghbanzad et al., [20] conducted experiment of hybrid silica nanosphere and multi wall carbon nanotube (MWCNT). They found that an increase in hybrid nanofluid thermal conductivity leads to enhancement of $23.3 \%$ MWCNT and $8.8 \%$ silica nanoparticle. Moreover, the use of $\mathrm{Al}_{2} \mathrm{O}_{3}$ $\mathrm{Cu} /$ water hybrid nanofluid in heat sink for application in cooling electronics has demonstrated increased in convective heat transfer compared to water [21]. Another innovative study was conducted by Abbasi and Rashidi [22] on thermal conductivity of hybrid multiwall carbon nanotube and gamma alumina. They reported an increase in thermal conductivity of $20.68 \%$ at volume concentration of $0.1 \%$. Baby et al., [23] considered hybrid nanofluid of hydrogen exfoliated graphene (HEG) and multi wall carbon nanotube (MWCNT) with convective heat transfer coefficient enhancement of around $570 \%$ at volume fraction of $0.005 \%$. Differently, Balla et al. [24] carried out numerical study of hybrid nanofluid of $\mathrm{CuO}-\mathrm{Cu}$ nanoparticles with heat transfer enhancement dependent on nanofluid Reynolds number increase. Convective heat transfer and effect of Nusselt number of hybrid Al2O3-Cu/water in circular tube has been explored by Suresh et al., [25]. The result revealed $13.56 \%$ enhancement of Nusselt number at Reynolds number of 1730 .

\section{Synthesis of Hybrid Nanoparticles}

Synthesis of nanoparticle is the first step for obtaining a good hybrid nanofluid. The synthesis methods of different hybrid nanoparticles are summarized below. 


\subsection{Synthesis of $\mathrm{y}-\mathrm{Al}_{2} \mathrm{O}_{3} / \mathrm{MWCNTS}$}

Pure MWCNT was functionalized by treating it with nitric acid. The nitric acid-MWCNT suspension was refluxed followed by stirring for $4 \mathrm{~h}$. The suspension was ultra-sonicated in ultrasonic water bath for $4 \mathrm{~h}$ at $600 \mathrm{c}$. The above sample is then washed in distilled water in order to obtain neutral $\mathrm{pH}$ and finally dried at $900 \mathrm{c}$ for $24 \mathrm{~h}$. Aluminium acetate powder was dissolved in ethanol under vigorous stirring at room temperature for $30 \mathrm{~min}$. The functionalized MWCNTs and pure MWCNT are then added to aluminium-ethanol suspension at room temperature with the aid of ultrasonicator and then placed under vacuum $(50 \mathrm{cmHg})$ for $24 \mathrm{~h}$. Ammonia solution was added slowly to the mixture to adjust the $\mathrm{pH}$ above 9 and thus obtain fine boehmite particles. The solution was then transferred to a $350 \mathrm{ml}$ Teflon-lined stainless steel auto- clave chamber, where the solvothermal synthesis was conducted. 16 bar pressure was maintained in the autoclave for the synthesis and the solution was kept for $24 \mathrm{~h}$ at $2000 \mathrm{c}$. The autoclave was allowed to cool to room temperature and the collected precipitate was washed thoroughly with ethanol to obtain a neutral $\mathrm{pH}$ and then vacuumdried at $60 \mathrm{cc}$ for $6 \mathrm{~h}$. The resulting powder is finally heated in argon atmosphere for $1 \mathrm{~h}$ at $5000 \mathrm{c}$. The hybrid nanofluid of $\mathrm{\gamma}$-AI2O3/MWCNTs is then finally obtained by dispersing the above hybrid nanopowder in deionized water which contain little amount of gum Arabic [22].

\subsection{Synthesis of Al2O3-Cu Nano-Composite Powder}

Soluble nitrates of copper $\left(\mathrm{Cu}\left(\mathrm{NO}_{3}\right)_{2} .3 \mathrm{H}_{2} \mathrm{O}\right)$ and aluminum, $\left(\mathrm{Al}\left(\mathrm{NO}_{3}\right)_{3} .9 \mathrm{H}_{2} \mathrm{O}\right)$ were dissolved in water. The proportions of the above salts were decided so as to have a predefined relative proportion of alumina and copper oxide in the powder mixture. The solution was spray dried at $180^{\circ} \mathrm{C}$ to obtain the precursor powder. The precursor powder was then heated at $900^{\circ} \mathrm{C}$ in air atmosphere for $60 \mathrm{~min}$ to form a powder mixture of copper oxide and stable $\mathrm{Al}_{2} \mathrm{O}_{3}$. A tubular furnace was used to heat the mixture at $400^{\circ} \mathrm{C}$ for 1 hour in hydrogen atmosphere. The powder sample was then placed in an alumina boat and then kept in a horizontally placed alumina tube of the furnace which was heated by silicon carbide heating elements. The $\mathrm{CuO}$ was preferentially reduced in hydrogen to metallic copper whereas $\mathrm{Al}_{2} \mathrm{O}_{3}$ remains unchanged. The powder mixture was finally ball milled at $400 \mathrm{rpm}$ for $1 \mathrm{hr}$. in order to obtain a homogeneous $\mathrm{Al}_{2} \mathrm{O}_{3}-\mathrm{Cu}$ nano-composite powder [26].

\subsection{Synthesis of $\mathrm{GO}-\mathrm{Fe}_{3} \mathrm{O}_{4}$}

Graphene oxide flakes were dissolved in $100 \mathrm{ml}$ of distilled water by ultra-sonication. $\mathrm{FeCl}_{3} \cdot 6 \mathrm{H}_{2} \mathrm{O}$ and $\mathrm{FeCl}_{2} .4 \mathrm{H}_{2} \mathrm{O}$ in a ratio of $1.75: 1$ were mixed with distilled water and the mixture was stirred with graphene oxide solution for $45 \mathrm{~min}$. sodium hydroxide was added in drop wise and a black precipitated was obtain. The precipitate was then washed with distilled water and finally freeze drying was done for $24 \mathrm{~h}$ to obtain $\mathrm{GO}-\mathrm{Fe}_{3} \mathrm{O}_{4}$ hybrid [27].

Hydrochloric acid and nitric acid in a molar ratio of 1:3 were mixed with CNT with the aid of magnetic stirrer for $72 \mathrm{~h}$ at $60^{\circ} \mathrm{C}$. The above mixture was washed with distilled water and acetone, followed by oven drying at $80^{\circ} \mathrm{C}$ for $24 \mathrm{~h}$. This process gives rise to the formation of carboxyl group within the CNT surface which facilitates its hybridization. Carboxylated-CNT was dispersed in $50 \mathrm{ml}$ of distilled water for $1 \mathrm{hr}$. by magnetic stirring, $\mathrm{FeCl}_{3}+\mathrm{FeCl}_{2}+$ salts in the molar ratio of 2:1 were added to the mixture. Aqueous sodium hydroxide was gradually added up to adjust the $\mathrm{pH}$ value to 12 , followed by stirring for $30 \mathrm{~min}$. Finally the precipitate is then washed with distilled water, acetone and dried for $24 \mathrm{~h}$ at $80^{\circ} \mathrm{C}$. 


\section{Preparation of Hybrid Nanofluid}

Hybrid nanofluids are generally prepared via single or two step method. Single step method is suitable for small scale production whereas two step method is cheap for mass production.

\subsection{Single-Step Method}

The single step method PWE (pulse wire evaporation) method it is most prominent method of producing nanofluid. The apparatus consists of a capacitor bank, a high-voltage DC power supply, a condensation chamber and a high-voltage gap switch. The process consists of directing high-voltage pulse (300V) through a thin wire and as a result of non-equilibrium heating within micro seconds the wire evaporates in to plasma. The plasma is then made in contact with inert gas $\operatorname{Ar}$ or $\mathrm{N}_{2}$ there by condensing the plasma in to nanosize powder. Nanofluid with given volume concentration is then poured in to an exploding bottle contained in the PWE instrument and then mixed with the above nanosize powder to obtain a hybrid nanofluid. This process is the most promising method of preparing low-cost nanofluid [28].

Lee et al., [29] reported that the size of nanoparticles depends on degree of super-heat applied to the wire: with increase in super heat, the particle size reduces. The particle size decrease with increase in pressure of the inert gas and the particle size decreases substantially with decrease in wire diameter. Munkhbayar et al. [30] utilized this process to prepare a hybrid nanofluid of silver/multi-walled carbon nano-tube (Ag/MWCNT). In their work MWCNT was purified using chemical process. Nitric acid and sulfuric acid where used to improve the exterior activity of the MWCNT as a result of its hydrophobic nature. Ag nanofluid was obtained using PWE method, the wire diameter used was $90 \mathrm{~mm}$ and the pulse-voltage was $300 \mathrm{~V}$. The concentrations of $\mathrm{Ag}$ nanoparticles were maintained in the hybrid composite by controlling the wire explosion number whereas the MWCNT was maintained at 0.05 w\%. Fig. 2 shows samples studied using X-ray diffractogram and Raman spectra of $f-M W C N T$, f-HEG and f-MWCNT+f-HEG respectively.

\subsection{Two-Step Method}

In this method the hybrid nanopowder is first of all produced via: chemical, physical or mechanical process such as grinding, milling, so - gel process or vapor phase method. Secondly the prepared hybrid nanopowder is then dispersed in to the base fluid by using high shear mixing equipment or ultra-sonication using ultrasonic vibrator. This process is very cheap for mass production of hybrid nanofluid. Baby and Sundara [31] utilized this method to produce a hybrid nanofluid containing silver and functionalize graphene. Chopkar et al., [32] used two step method to prepare a hybrid nanofluid of $\mathrm{Al}_{2} \mathrm{Cu}$ and $\mathrm{Ag}_{2} \mathrm{Al}$ with ethylene glycol and $\mathrm{DI}$ as base fluids. The composite powders were obtained using mechanical alloying. Fig. 3 shows the schematic of synthesis of CUO/HEG and making nanofluid with the composite. The HEG undergoes acidic treatment in order to introduce carboxyl and hydroxyl functional group on the graphene, the functionalize graphene was used to decorate $\mathrm{CuO}$ nanoparticles.

Chen et al., [33] utilized this method in the preparation of $\mathrm{Fe}_{2} \mathrm{O}_{3} / \mathrm{MWCNTs}$ nanofluid with sodium dodecyl-benzesulfonate (NaDDBS) as surfactant. Baby and Ramaprubhu [34] used two step method to prepare a hybrid nanofluid of copper oxide/graphene (CuO/HEG) with ethylene glycol and DI water as the base fluid. The copper oxide was synthesized using chemical reduction followed by calcinations at considerably low temperature whereas graphene was synthesized via hydrogen induced 
exfoliation. Suresh et al., [35] prepared Al2O3/Cu hybrid nanofluid via two step method with sodiumlauryl-sulphate (SLS) as dispersant.

\section{Factors Affecting Thermal Performance of Hybrid Nanofluid}

Hybrid nanofluid as a new type of fluid has good thermal characteristics. Stability is one of the main characteristics that influence its performance.

\subsection{Stability of Hybrid Nanofluids}

Stability is one of the critically factor that affect the performance of hybrid nanofluid [36]. The effect of stability of hybrid $\mathrm{Al}_{2} \mathrm{O}_{3}-\mathrm{Cu}$ nano composite was investigated at different volume fraction [37]. Hybrid nanofluids can lose their potential to transfer heat due to their proneness to coagulation. Thus, stability evaluation and investigation cannot be ignored. Lack of good stability can alter the thermo physical properties of hybrid nanofluids and will result in low thermal performance in heat transfer applications. Researcher have developed several method for stability analysis such as, Centrifugation method, Sedimentation method, Zeta potential analysis, Spectral analysis method, Electron microscopy and light scattering methods.

Combustion of fossil fuels in thermal power plant is a well-known method to generate electricity. The United States Energy Information Administration reports that fossil fuels were responsible for $66.8 \%$ of total global generated electricity in 2009 [19]. Diesel power plants comprise of diesel engines and other support systems typical of any power plant [5]. This power plant convert fossil fuel to be electrical energy [1]. The oil and gas engines are called Internal Combustion (IC) engines. The fuel burn inside the engines and the product of the combustion form the working fluid that generates mechanical power [6]. Several countries used diesel power plant to meet their energy demand such as India, Nigeria and Pakistan [2, 7-10]. As example, total generation of electricity produced by diesel power plant in India is $1022.39 \mathrm{MW}$ [7].

\section{2 pH Control of Hybrid Nanofluids}

Stability of nanofluid is directly related to its electro-kinetic properties; therefore, $\mathrm{pH}$ control can increase stability due to strong repulsive forces. Simple acid treatment could cause good stability of CNT in water [38]. Fovet et al., [39] investigated various $\mathrm{pH}$ values for Al2O3 nano-fluid and observed changes in agglomeration by altering the $\mathrm{pH}$ value. More so, $\mathrm{pH}$ value differs from one sample to another. For instance, suitable $\mathrm{pH}$ value for alumina, copper and graphite dispersed in water are around $8,9.5$ and 2, respectively [40].

\subsection{Effect of Ultrasound Intensity}

The intensity of ultra-sonication plays an important role in altering the morphological and dimensional characteristics of nanoparticles. Increasing the ultrasound intensity increases the phenomenal cavitations so the collapsed cavity in the solution creates a shockwave within the solution there by reducing the particles size and enhancing the stability of the nanofluid [41]. 


\section{Thermal Performance of Hybrid Nanofluid}

Many researchers have conducted studies on thermal conductivity enhancement using hybrid nanofluid. KD2 pro thermal analyzer which operates on modified transient hot wire equipment was used by most of the researchers.

Chopkar et al., [32] synthesized $\mathrm{Al}_{2} \mathrm{Cu}$ and $\mathrm{Ag}_{2} \mathrm{Al}$ via mechanical alloying and study the thermal conductivities of the hybrid nanofluids. At $1 \%$ volume fraction of $\mathrm{Al}_{2} \mathrm{Cu} /$ deionize water base nanofluid $70 \%$ enhancement in thermal conductivity was obtained in comparison to base fluid and $50 \%$ was obtained for ethylene glycol base hybrid nanofluid. Base on this work the thermal conductivity increases with increase in volume fraction. Plate like shape hybrid nanoparticles and cylindrical shapes gives better enhancement than spherical shape nanoparticles. A high enhancement was recorded for $\mathrm{Ag}_{2} \mathrm{Al}$ water base nanofluid in comparison with $\mathrm{Al}_{2} \mathrm{Cu}$ nanofluid and the enhancement is as a result of higher thermal conductivity of silver than copper.

Jana and Zhong [42] conducted an experiment for thermal conductivity enhancement of carbon nanotubes (CNTs), copper nanoparticles (CuNPs), gold nanoparticles (AuNPs) and their hybrids. Base on this experiment $34 \%$ enhancement in thermal conductivity was obtain at $0.8 \%$ volume fraction of CNT, the normalize thermal conductivity was nonlinearly dependent on volume fraction of CNT and the non-linearity may be due to the size, shape and loading of CNT in the nanofluid. For CuNP suspension the normalize thermal conductivity increases with increase in CuNP volume fraction,74\% increment in thermal conductivity over water was obtain at room temperature, this high increment in comparison with $40 \%$ enhancement obtain for ethylene glycol base fluid is as a result of size of the Cu nanoparticles ( $35-50 \mathrm{~nm}$ ) and better dispersion due to instant bath sonication. It was observed that at volume fraction of $1.4 \%$ AuNP, $37 \%$ increment in thermal conductivity was obtained over water.

Hybrid nanofluid of silver/functionalize graphene (Ag/HEG) was prepared by Baby and Sundara [31] with deionize water and ethylene glycol as base fluid. From this experiment, it was found that the thermal conductivity of the hybrid nanofluids increases with increase in temperature and volume concentrations. $7 \%$ enhancement was obtained at temperature of $250 \mathrm{c}$ and $13 \%$ at $700 \mathrm{c}$ for $0.005 \%$ volume fraction of $(\mathrm{Ag} / \mathrm{HEG})$ deionize water nanofluid. As the volume fraction increases to $0.05 \%$ the enhancements were $25 \%$ and $86 \%$ at temperature of $250 \mathrm{c}$ and $700 \mathrm{c}$ respectively. On the other hand, ethylene glycol base nanofluid shows an enhancement, but not as for deionize water. The enhancement was firstly obtained at volume fraction of $0.05 \%$ un like the first one, the low thermal conductivity enhancement was due to high viscosity of the base fluid.

From the work of Baby and Ramaprubhu [34] Copper oxide was decorated with graphene; the graphene was obtained via reduction of graphene oxide. (CuO/HEG) was dispersed in deionize water and ethylene glycol in a volume fraction of $0.05 \%$ and $0.01 \%$. At $0.05 \%$ volume fraction $28 \%$ enhancement was obtain in thermal conductivity at $250 \mathrm{c}$ and $90 \%$ was obtain at $500 \mathrm{c}$ this increment can be due to high thermal conductivity of copper oxide and graphene. With increase in volume fraction the particle - particle distance decreases, thus, due to percolation effect more particle is in contact with each other the frequency of lattice vibration increases. The enhancement in thermal conductivity of CuO/HEG dispersed in ethylene glycol based nanofluid was not as high as that of deionize water base nanofluid. $17 \%$ enhancement was obtained at $250 \mathrm{c}$ for a volume fraction of $0.07 \%$ and $23 \%$ was obtain at 50 oc. the enhancement was not high as expected this could be due to the formation of sp3 defects formed in the graphene sheet.

Thermal conductivity enhancement of Graphene and graphene multi - walled carbon nano-tube (MWCNTs) was conducted by Aravind and Ramaprabhu [43]. It was observed base on this experiment that the thermal conductivity increases with increase in volume fraction, they obtain $9.2 \%$ and $73 \%$ 
for volume fraction of $0.04 \%$ at temperature of $250 \mathrm{c}$ and $500 \mathrm{c}$ respectively for deionize water base nanofluid. $6.9 \%$ and $20 \%$ was obtained for ethylene glycol at the same temperature and volume fraction respectively. For the composite nanofluid that is graphene - multi-walled carbon nano-tubes they obtain $10.5 \%$ and $87.9 \%$ for volume fraction of 0.04 at temperature of 250 c and 500 c respectively for deionize water base nanofluid. For ethylene glycol, 13.7 and $24 \%$ was recorded at the same volume fraction and temperature respectively.

Iron oxide/multi-walled carbon nanotube ( $\mathrm{Fe}_{2} \mathrm{O}_{3} / \mathrm{MWCNTs}$ ) nanofluid was synthesized by Chen et al., [33] and the thermal conductivity of the hybrid nanofluid was evaluated by varying the volume concentration of the iron oxide in the hybrid nanofluid. $27.7 \%$ enhancement in thermal conductivity was obtained with 0.02 wt $\% \mathrm{Fe}_{2} \mathrm{O}_{3}$ and $0.05 \%$ MWCNTs, the enhancement was due to Iron oxide $\left(\mathrm{Fe}_{2} \mathrm{O}_{3}\right)$ aggregate locally on MWCNTs surface their by forming chains along the carbon nanotubes. It was observed based on this experiment, increase in concentration of $\mathrm{Fe}_{2} \mathrm{O}_{3}$ nanoparticles greater than $0.02 \%$, the thermal conductivity decreases. This is due to excess aggregation of the nanoparticles personally which confine the formation of effective heat transfer networks.

Aluminum oxide/copper $\left(\mathrm{Al}_{2} \mathrm{O}_{3} / \mathrm{Cu}\right)$ nanofluid shows a linear relation in thermal conductivity enhancement with increase in volume fraction. The thermal conductivity enhancement of the hybrid nanofluid was compared with the enhancements shown by alumina/water nanofluids. Base on the comparison, there is a very significant enhancement in the effective thermal conductivity due to the hybridization of alumina nanoparticles using metallic copper particles. However, it has been found that viscosity increase substantially higher than the thermal conductivity.

\subsection{Heat Transfer Characteristics of Hybrid Nanofluid}

Moghadassi et al., [44] conducted a numerical study on heat transfer characteristics of Al2O3/Cu hybrid nanofluid. The simulated result shows that the Nusselt number and heat transfer coefficient increases with Reynolds number. The pressure drop and the friction factor coefficient were observed to increase with increase in volume concentration.

Labib et al., [45] numerically studied the force convective heat transfer of $\mathrm{CNT} / \mathrm{Al}_{2} \mathrm{O}_{3}$ nanofluid, based on their study the convective heat transfer performance was observe to increase significantly. The increment was due to higher shear thinning behaviour of the CNT which causes thinner boundary layer.

From the work of Abbasi et al., [22], a greater stability and thermal conductivity was obtained on $\mathrm{Y}-\mathrm{Al}_{2} \mathrm{O}_{3} / \mathrm{MWCNTS}$ nanofluid with low concentration of carboxylic-acid group than the hybrid nanofluid which contains higher value. It was further gathered that treating nanoparticles with relatively higher straightness ratio would cause excessive deterioration of the aspect ratio, thereby decreasing the thermal conductivity of the hybrid nanofluid.

\section{Conclusion}

In conclusion, the process of preparation, factors affecting the performance of hybrid nanofluid have been extensively discussed. Meanwhile, in this review the thermal characteristics of hybrid nanofluid were found to be higher in comparison to the base fluid and fluid containing single nanoparticles respectively. It was also observed that the characteristics of hybrid nanofluid increases with increase in temperature and volume fraction, whereas for some hybrid nanofluid there are upper limits for the volume fraction such that the performance deteriorates at higher volume concentration. 
This work focuses on preparation and thermal characteristics of hybrid nanofluids, however further research is required for better understanding of the characteristics of the fluid. More experimental studies are required for the best method that will suit each hybrid nanofluid preparation in terms of performance, because different methods yield different result. In terms of compatibility more experiments are required in order to identify the nanoparticles that are compatible. Further research need to focus in finding the upper limits in terms of volume fraction and the ratio of the nanoparticles in the composite powder.

\section{References}

[1] Lee, S., SU-S. Choi, S, and Li, and J. A. Eastman. "Measuring thermal conductivity of fluids containing oxide nanoparticles." (1999): 280-289. https://doi.org/10.1115/1.2825978

[2] Khattak, M. A., A. Mukhtar, and S. Kamran Afaq. "Application of nano-fluids as coolant in heat exchangers: a review." J. Adv. Rev. Sci. Res 22, no. 1 (2016): 1-11.

[3] Sidik, NA Che, and O. Adnan Alawi. "Computational investigations on heat transfer enhancement using nanorefrigerants." J. Adv. Res. Des. 1, no. 1 (2014): 35-41.

[4] Lee, Y. K. "The use of nanofluids in domestic water heat exchanger." J. Adv. Res. Appl. Mech 3, no. 1 (2014): 9-24.

[5] Turcu, R., A. L. Darabont, A. Nan, N. Aldea, D. Macovei, D. Bica, L. Vekas et al. "New polypyrrole-multiwall carbon nanotubes hybrid materials." Journal of Optoelectronics and Advanced Materials 8, no. 2 (2006): 643-647.

[6] Jana, Soumen, Amin Salehi-Khojin, and Wei-Hong Zhong. "Enhancement of fluid thermal conductivity by the addition of single and hybrid nano-additives." Thermochimica acta 462, no. 1-2 (2007): 45-55. https://doi.org/10.1016/i.tca.2007.06.009

[7] Turcu, R., A. Nan, I. Craciunescu, S. Karsten, O. Pana, I. Bratu, D. Bica et al. "Functionalized nanostructures with magnetic core and pyrrole copolymers shell." (2007).

[8] Yen, T. H., C. Y. Soong, and P. Y. Tzeng. "Hybrid molecular dynamics-continuum simulation for nano/mesoscale channel flows." Microfluidics and Nanofluidics 3, no. 6 (2007): 665-675. https://doi.org/10.1007/s10404-007-0154-7

[9] "Air and Waste Management Association - 100th Annual Conference and Exhibition of the Air and Waste Management Association 2007," in ASME International Mechanical Engineering Congress and Exposition, Proceedings, 2008.

[10] Turcu, R., A. Nan, I. Craciunescu, J. Liebsher, O. Pana, D. Bica, L. Vekas, and C. Mijangos. "Comparative study of hybrid nanostructures of polymermagnetic nanoparticles." J Optoelectron Adv Mater 10 (2008): 2237-2243.

[11] Turcu, R., O. Pana, A. Nan, I. Craciunescu, O. Chauvet, and C. Payen. "Polypyrrole coated magnetite nanoparticles from water based nanofluids." Journal of Physics D: Applied Physics 41, no. 24 (2008): 245002. https://doi.org/10.1088/0022-3727/41/24/245002

[12] Jha, Neetu, and S. Ramaprabhu. "Thermal conductivity studies of metal dispersed multiwalled carbon nanotubes in water and ethylene glycol based nanofluids." Journal of Applied Physics 106, no. 8 (2009): 084317. https://doi.org/10.1063/1.3240307

[13] Ho, C. J., J. B. Huang, P. S. Tsai, and Y. M. Yang. "On laminar convective cooling performance of hybrid water-based suspensions of Al2O3 nanoparticles and MEPCM particles in a circular tube." International Journal of Heat and Mass Transfer 54, no. 11-12 (2011): 2397-2407. https://doi.org/10.1016/i.ijheatmasstransfer.2011.02.022

[14] Suresh, S., K. P. Venkitaraj, P. Selvakumar, and M. Chandrasekar. "Synthesis of Al2O3-Cu/water hybrid nanofluids using two step method and its thermo physical properties." Colloids and Surfaces A: Physicochemical and Engineering Aspects 388, no. 1-3 (2011): 41-48. https://doi.org/10.1016/i.colsurfa.2011.08.005

[15] Baby, Tessy Theres, and Sundara Ramaprabhu. "Experimental investigation of the thermal transport properties of a carbon nanohybrid dispersed nanofluid." Nanoscale 3, no. 5 (2011): 2208-2214. https://doi.org/10.1039/c0nr01024c

[16] Aspnes, David E. "Optical properties of thin films." Thin solid films 89, no. 3 (1982): 249-262. https://doi.org/10.1016/0040-6090(82)90590-9

[17] Jamil, M., N. C. Sidik, and MNAW Muhammad Yazid. "Thermal performance of thermosyphon evacuated tube solar collector using TiO2/water nanofluid." J. Adv. Res. Fluid Mech. Therm. Sci 20, no. 1 (2016): 12-29.

[18] Azwadi, CS Nor, and I. M. Adamu. "Turbulent force convective heat transfer of hybrid nano fluid in a circular channel with constant heat flux." J. Adv. Res. Fluid Mech. Therm. Sci 19, no. 1 (2016): 1-9. 
[19] Noh, N. M., A. Fazeli, and NA Che Sidik. "Numerical simulation of nanofluids for cooling efficiency in microchannel heat sink." J. Adv. Res. Fluid Mech. Therm. Sci 4, no. 1 (2014): 13-23.

[20] Baghbanzadeh, Mohammadali, Alimorad Rashidi, Davood Rashtchian, Roghayeh Lotfi, and Azadeh Amrollahi. "Synthesis of spherical silica/multiwall carbon nanotubes hybrid nanostructures and investigation of thermal conductivity of related nanofluids." Thermochimica acta 549 (2012): 87-94. https://doi.org/10.1016/i.tca.2012.09.006

[21] Baghbanzadeh, Mohammadali, Alimorad Rashidi, Davood Rashtchian, Roghayeh Lotfi, and Azadeh Amrollahi. "Synthesis of spherical silica/multiwall carbon nanotubes hybrid nanostructures and investigation of thermal conductivity of related nanofluids." Thermochimica acta 549 (2012): 87-94. https://doi.org/10.1016/i.tca.2012.09.006

[22] Abbasi, Saloumeh Mesgari, Alimorad Rashidi, Ali Nemati, and Kaveh Arzani. "The effect of functionalisation method on the stability and the thermal conductivity of nanofluid hybrids of carbon nanotubes/gamma alumina." Ceramics International 39, no. 4 (2013): 3885-3891. https://doi.org/10.1016/j.ceramint.2012.10.232

[23] Theres Baby, Tessy, and Ramaprabhu Sundara. "Synthesis of silver nanoparticle decorated multiwalled carbon nanotubes-graphene mixture and its heat transfer studies in nanofluid." AIP Advances 3, no. 1 (2013): 012111. https://doi.org/10.1063/1.4789404

[24] Balla, Hyder H., Shahrir Abdullah, Wan MohdFaizal, Rozli Zulkifli, and Kamaruzaman Sopian. "Numerical study of the enhancement of heat transfer for hybrid CuO-Cu nanofluids flowing in a circular pipe." Journal of oleo science 62, no. 7 (2013): 533-539. https://doi.org/10.5650/jos.62.533

[25] Suresh, S., K. P. Venkitaraj, P. Selvakumar, and M. Chandrasekar. "Effect of Al2O3-Cu/water hybrid nanofluid in heat transfer." Experimental Thermal and Fluid Science 38 (2012): 54-60.

https://doi.org/10.1016/i.expthermflusci.2011.11.007

[26] Suresh, S., K. P. Venkitaraj, and P. Selvakumar. "Synthesis, Characterisation of Al2O3-Cu Nano composite powder and water based nanofluids." In Advanced Materials Research, vol. 328, pp. 1560-1567. Trans Tech Publications Ltd, 2011. https://doi.org/10.4028/www.scientific.net/AMR.328-330.1560

[27] Sundar, L. Syam, Manoj K. Singh, and Antonio CM Sousa. "Thermal conductivity of ethylene glycol and water mixture based Fe304 nanofluid." International communications in heat and mass transfer 49 (2013): 17-24. https://doi.org/10.1016/j.icheatmasstransfer.2013.08.026

[28] Lee, Geun Hee, Joong Hak Park, Chang Kyu Rhee, and Whung Whoe Kim. "Fabrication of Al nano powders by pulsed wire evaporation (PWE) method." Journal of Industrial and Engineering Chemistry 9, no. 1 (2003): 71-75.

[29] Lee, P. Y., K. Ishizaka, H. Suematsu, W. Jiang, and K. Yatsui. "Magnetic and gas sensing property of nanosized NiFe 204 powders synthesized by pulsed wire discharge." Journal of Nanoparticle Research 8, no. 1 (2006): 29. https://doi.org/10.1007/s11051-005-5427-z

[30] Munkhbayar, B., Md Riyad Tanshen, Jinseong Jeoun, Hanshik Chung, and Hyomin Jeong. "Surfactant-free dispersion of silver nanoparticles into MWCNT-aqueous nanofluids prepared by one-step technique and their thermal characteristics." Ceramics International 39, no. 6 (2013): 6415-6425. https://doi.org/10.1016/i.ceramint.2013.01.069

[31] Baby, Tessy Theres, and Sundara Ramaprabhu. "Synthesis and nanofluid application of silver nanoparticles decorated graphene." Journal of Materials Chemistry 21, no. 26 (2011): 9702-9709.

https://doi.org/10.1039/c0jm04106h

[32] Chopkar, Manoj, S. Kumar, D. R. Bhandari, Prasanta K. Das, and Indranil Manna. "Development and characterization of Al2Cu and Ag2Al nanoparticle dispersed water and ethylene glycol based nanofluid." Materials Science and Engineering: B 139, no. 2-3 (2007): 141-148. https://doi.org/10.1016/j.mseb.2007.01.048

[33] Chen, Li Fei, Min Cheng, De Jun Yang, and Lei Yang. "Enhanced thermal conductivity of nanofluid by synergistic effect of multi-walled carbon nanotubes and Fe2O3 nanoparticles." In Applied Mechanics and Materials, vol. 548, pp. 118-123. Trans Tech Publications Ltd, 2014. https://doi.org/10.4028/www.scientific.net/AMM.548-549.118

[34] Baby, Tessy Theres, and Ramaprabhu Sundara. "Synthesis and transport properties of metal oxide decorated graphene dispersed nanofluids." The Journal of Physical Chemistry C 115, no. 17 (2011): 8527-8533. https://doi.org/10.1021/ip200273g

[35] Suresh, S., K. P. Venkitaraj, P. Selvakumar, and M. Chandrasekar. "Effect of Al2O3-Cu/water hybrid nanofluid in heat transfer." Experimental Thermal and Fluid Science 38 (2012): 54-60.

https://doi.org/10.1016/i.expthermflusci.2011.11.007 
[36] Hwang, Yu-jin, J. K. Lee, C. H. Lee, Y. M. Jung, S. I. Cheong, C. G. Lee, B. C. Ku, and S. P. Jang. "Stability and thermal conductivity characteristics of nanofluids." Thermochimica Acta 455, no. 1-2 (2007): 70-74. https://doi.org/10.1016/i.tca.2006.11.036

[37] Suresh, S., K. P. Venkitaraj, and P. Selvakumar. "Synthesis, Characterisation of Al2O3-Cu Nano composite powder and water based nanofluids." In Advanced Materials Research, vol. 328, pp. 1560-1567. Trans Tech Publications Ltd, 2011. https://doi.org/10.4028/www.scientific.net/AMR.328-330.1560

[38] Wen, Dongsheng, Guiping Lin, Saeid Vafaei, and Kai Zhang. "Review of nanofluids for heat transfer applications." Particuology 7, no. 2 (2009): 141-150. https://doi.org/10.1016/i.partic.2009.01.007

[39] Fovet, Yannick, Jean-Yves Gal, and Florence Toumelin-Chemla. "Influence of $\mathrm{pH}$ and fluoride concentration on titanium passivating layer: stability of titanium dioxide." Talanta 53, no. 5 (2001): 1053-1063. https://doi.org/10.1016/S0039-9140(00)00592-0

[40] Xie, Huaqing, Hohyun Lee, Wonjin Youn, and Mansoo Choi. "Nanofluids containing multiwalled carbon nanotubes and their enhanced thermal conductivities." Journal of Applied physics 94, no. 8 (2003): $4967-4971$. https://doi.org/10.1063/1.1613374

[41] Chattopadhyay, Pratibhash, and Ram B. Gupta. "Production of griseofulvin nanoparticles using supercritical CO2 antisolvent with enhanced mass transfer." International journal of pharmaceutics 228, no. 1-2 (2001): 19-31. https://doi.org/10.1016/S0378-5173(01)00803-1

[42] Jana, Soumen, Amin Salehi-Khojin, and Wei-Hong Zhong. "Enhancement of fluid thermal conductivity by the addition of single and hybrid nano-additives." Thermochimica acta 462, no. 1-2 (2007): 45-55. https://doi.org/10.1016/i.tca.2007.06.009

[43] Aravind, SS Jyothirmayee, and S. Ramaprabhu. "Graphene-multiwalled carbon nanotube-based nanofluids for improved heat dissipation." Rsc Advances 3, no. 13 (2013): 4199-4206. https://doi.org/10.1039/c3ra22653k

[44] Moghadassi, Abdolreza, Ehsan Ghomi, and Fahime Parvizian. "A numerical study of water based Al2O3 and Al2O3Cu hybrid nanofluid effect on forced convective heat transfer." International Journal of Thermal Sciences 92 (2015): 50-57. https://doi.org/10.1016/j.ijthermalsci.2015.01.025

[45] Labib, M. Nuim, Md J. Nine, Handry Afrianto, Hanshik Chung, and Hyomin Jeong. "Numerical investigation on effect of base fluids and hybrid nanofluid in forced convective heat transfer." International Journal of Thermal Sciences 71 (2013): 163-171. https://doi.org/10.1016/j.ijthermalsci.2013.04.003 